\title{
Axonal Transport of Noradrenaline, Protein and Glycoprotein in Cat Hypogastric Nerves in Vitro Under Conditions of High Extracellular Glucose
}

\author{
D. R. Tomlinson \\ Department of Physiology and Pharmacology, The Medical School, Queen's Medical Centre, Nottingham, UK
}

\begin{abstract}
Summary. Inferior mesenteric ganglia with attached hypogastric nerves were removed from anaesthetised cats and maintained in Eagle's Minimal Essential Medium in specially designed organ culture chambers for $48 \mathrm{~h}$ at $37^{\circ} \mathrm{C}$. The chambers had removable barriers so that the fluid bathing the ganglia was separated from that bathing the nerve trunks by inert silicone grease seals. $\left({ }^{3} \mathrm{H}\right)$-fucose $(1.25 \mu \mathrm{Ci} / \mathrm{ml})$ and $\left({ }^{14} \mathrm{C}\right)$-leucine $(0.5 \mu \mathrm{Ci} / \mathrm{ml})$ were added to the fluid bathing the ganglia at the start of the experiment. At this time, tight ligatures were applied to the hypogastric nerves so that the incorporated isotopes accumulated proximal to the constrictions due to interrupted axonal transport. The nerve trunks were bathed in medium containing glucose at either 5.5 or $25 \mathrm{mmol} / 1$. At the end of the incubation period, the accumulations, proximal to the constrictions, of incorporated leucine and fucose and of noradrenaline were measured. The high glucose medium caused
\end{abstract}

a $27.5 \%(p<0.001)$ reduction in the accumulation of fucose and a $19.2 \%(p<0.01)$ reduction in the accumulation of leucine. The incorporation of these tracers in the ganglia was unaffected by exposure of the nerve trunks to high glucose. The accumulation of noradrenaline proximal to the constrictions was also unaffected. Further experiments were performed to show that the reduced accumulations of fucose and leucine under conditions of high glucose were not due to loss of isotope from macromolecules in the axons nor to the small increase in osmotic pressure effected by addition of extra glucose to the culture medium. It is therefore suggested that elevation of extracellular glucose caused an impairment of axonal transport of incorporated leucine and fucose.

Key words: Diabetic neuropathy, cat, axonal transport, noradrenaline, nerve crush, glucose.
Nerve terminals are dependent for survival on the delivery of materials, synthesised in the cell body, by axonal transport. Impairment of this process causes degeneration and loss of function of the distal portions of the neurone $[5,17]$. It is, therefore, possible that disorders of axonal transport could contribute to the development of neuropathies in certain disease states.

Several groups of workers have shown that impairment of axonal transport of various macromolecules occurs within a few weeks or even days after induction of experimental diabetes [8-11, 14-16, 18-21, 24]. It has been postulated that the impairment of axonal transport may arise from persistent hyperglycaemia rather than some other feature of the diabetic state [21]. The experiments described here were performed to test this hypothesis.

Axonal transport was studied in vitro by measurement of the accumulation of materials proximal to a constriction applied to cat hypogastric nerves attached to the inferior mesenteric ganglion maintained in organ culture [2]. The effects of exposure of the axons to culture medium containing an elevated glucose concentration were studied.

\section{Materials and Methods}

\section{Single Ligation Experiments}

A preparation comprising the inferior mesenteric ganglion with attached hypogastric nerves, ligated $2 \mathrm{~cm}$ distal to the ganglion, was removed from anaesthetised cats (weight: $2.5-3.5 \mathrm{~kg}$ ), which had received a normal diet, using an aseptic technique as described elsewhere [2]. The preparation was transferred immediately to either a two or three compartment culture chamber (Fig.1). In the two compartment chambers the preparation was positioned so that the ganglion and $4 \mathrm{~mm}$ of the nerve trunks were located on one side of the barrier whilst the remaining nerve trunks, $1.5 \mathrm{~cm}$ in length, occupied the other compartment. With the three compartment chambers, one nerve passed through each barrier giving $1.5 \mathrm{~cm}$ of nerve trunk in each of the outer compartments with the ganglion in the centre. In all cases, nerve trunks were sealed to the barriers with inert silicone grease thereby separating the fluids on each side. 
a
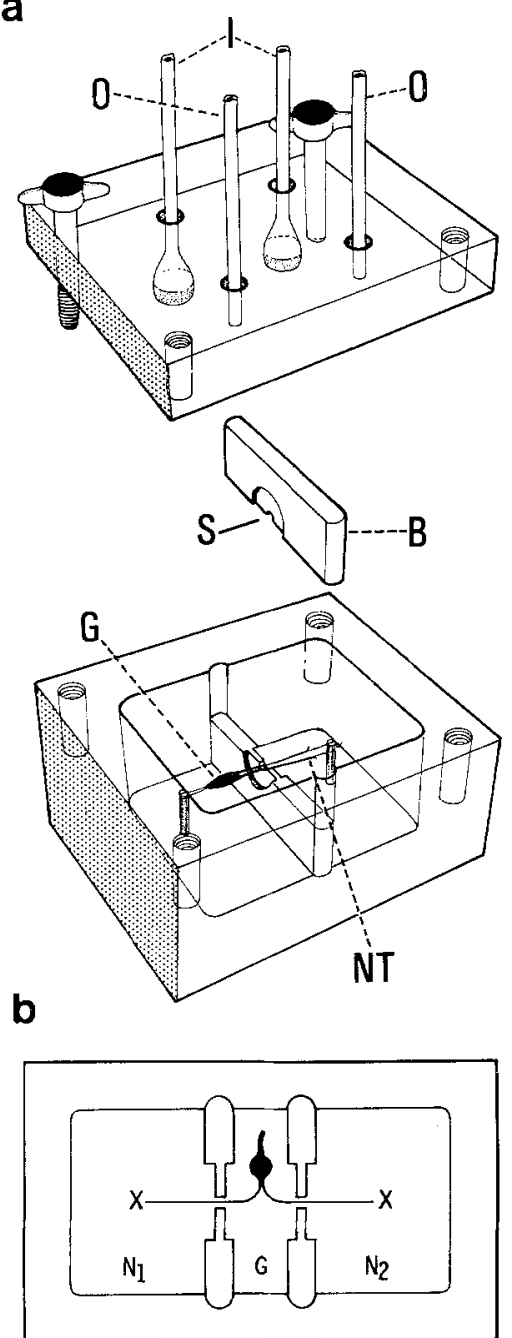

Fig. $1 \mathrm{a}$ and b. a Diagram illustrating the design of the twin-compartment culture chamber. A ganglion $(G) /$ nerve $(N T)$ preparation is shown in position with the nerve trunks resting in the 'hemi-port' of the lower half of the barrier. The upper half of the barrier $(B)$ is slid into position so that the upper 'hemi-port', filled with grease $(S)$, seals around the nerve trunks without crushing them. Culture media and radioisotopes are added and the lid is secured. The gas inlet tubes $(I)$ are connected to filtered, humidified $95 \% \mathrm{O}_{2}$ and $5 \% \mathrm{CO}_{2}$. The gas escapes via the outlet tubes $(O)$. b Plan view of the three-compartment culture chamber. The basic design is identical to the one above except that two barriers are incorporated to permit, for instance, incubation of one nerve trunk $\left(N_{l}\right)$ in $5.5 \mathrm{mmol} / 1$ glucose and the other $\left(N_{2}\right)$ in $25 \mathrm{mmol} / \mathrm{g}$ glucose. The ganglion $(G)$ is maintained in $5.5 \mathrm{mmol} / 1 \mathrm{glu}-$ cose

All compartments in both types of chamber contained Eagle's Minimal Essential culture medium (MEM) (Flow Laboratories, Ayrshire, Scotland) gassed with $95 \% \mathrm{O}_{2}$ and $5 \% \mathrm{CO}_{2}$ which was passed through a bacteriostatic filter and a sterile humidifier before entering the chambers. The composition of this medium was exactly as is described in Eagle's original paper [6]. Penicillin $(100 \mathrm{U} / \mathrm{ml})$ and streptomycin $(100 \mu \mathrm{g} / \mathrm{ml})$ were added to the culture medium. Selective increases in the glucose content of the medium were made as follows. In control experiments using the twin compartment chambers, the Dglucose in both compartments was left at the normal concentration for Eagle's MEM of $5.5 \mathrm{mmol} / 1$. In other experiments with these chambers, the D-glucose concentration of the fluid bathing the nerve trunks only was elevated to $25 \mathrm{mmol} / 1$. In experiments using the three compartment chambers, the D-glucose concentration of the fluid bathing one nerve trunk was left at $5.5 \mathrm{mmol} / 1$, whilst that bathing the other nerve trunk was raised to $25 \mathrm{mmol} / 1$.

Experiments were also performed to make distinction between the effects of elevated D-glucose per se and effects attributable to the increase in osmolality effected by the addition of an extra $19.5 \mathrm{mosmol}$ to the culture medium. These experiments used the three compartment chambers exactly as described above except that the extra Dglucose was added with sufficient water to maintain normal osmotic pressure in the final medium.

In all experiments $\left({ }^{3} \mathrm{H}\right)$-fucose and $\left({ }^{14} \mathrm{C}\right)$-leucine (Amersham International, Buckinghamshire, $\mathrm{UK}$ ) were added to the fluid bathing the ganglia at concentrations of 1.25 and $0.5 \mu \mathrm{Ci} / \mathrm{ml}$. The culture chambers were kept in a $37^{\circ} \mathrm{C}$ incubator for $48 \mathrm{~h}$.

\section{Sample Processing}

At the end of the incubation and before unsealing the barriers, samples of fluid were removed from all compartments for liquid scintillation counting and glucose assay. The latter was performed by a glucose oxidase method using 4-aminophenazone with phenol as a chromophore. In practice the method was used in the form of a specific test kit ('GOD-PAP method'; Boehringer Corporation, London), treating samples of medium exactly as if they were plasma.

The preparations were then removed from the boxes and washed in three changes of fresh culture medium to remove surplus radioactivity. The preparations were then placed on an aluminium block cooled to $4{ }^{\circ} \mathrm{C}$ for dissection. The $10 \mathrm{~mm}$ of nerve trunk proximal to each constriction were cut into $1 \mathrm{~mm}$ segments under a dissection microscope. These segments were transferred to $0.1 \mathrm{ml}$ ice-cold $5 \%$ perchloric acid. The ganglia were dissected free of connective tissue and nerve trunks. These were then blotted three times with filter paper, weighed quickly and each placed in $0.1 \mathrm{ml}$ ice-cold $5 \%$ perchloric acid. Nerve segments and ganglia were then homogenised by hand and centrifuged at $9,000 \mathrm{~g}$ for $20 \mathrm{~min}$. The supernatants were removed, the pellets re-extracted with a further $0.1 \mathrm{ml}$ cold $5 \%$ perchloric acid, recentrifuged and the two supernatants from each sample pooled. The pellets were then dissolved ( $24 \mathrm{~h}$ at room temperature) in NCS tissue solubilizer (Amersham International, Buckinghamshire, UK).

Radioactivity in the dissolved perchlorate precipitates and in $50 \mu \mathrm{l}$ of each perchlorate extract was measured by dual isotope liquid scintillation counting.

The remainder of the perchlorate extracts was used to measure the noradrenaline contents after dilution with $1 \mathrm{~N} \mathrm{NaOH}$ to increase the $\mathrm{pH}$ and to give a volume adequate for duplicate assays. This was done using high-performance liquid chromatography with electrochemical detection $[7,23]$.

\section{Double Ligation Experiments}

The findings from the experiments described above (see Results) indicated the need for a series of experiments designed to examine the effects of high glucose on materials which had already accumulated under conditions of normal glucose. These experiments employed the three compartment chambers. Preparations were set up in these exactly as described above except that the glucose concentration in all three compartments was $5.5 \mathrm{mmol} / \mathrm{l}$. These preparations were incubated for $24 \mathrm{~h}$. At the end of this time the chambers were opened and a second ligature applied to each nerve $5 \mathrm{~mm}$ proximal to the first. The culture medium in the outer two compartments was then changed. In one compartment was placed fresh medium containing $5.5 \mathrm{mmol} / \mathrm{l} \mathrm{glu}-$ cose, in the other the medium contained $25 \mathrm{mmol} / 1$ glucose. The preparations were then incubated for a further $24 \mathrm{~h}$. In this way, the effects of the high glucose could be manifest both on material which had already accumulated during the first $24 \mathrm{~h}$ in conditions of normal glucose as well as on material which was accumulating during the second $24 \mathrm{~h}$ in conditions of high glucose.

In these experiments noradrenaline assays were not performed. 
Table 1. Accumulations proximal to constrictions in preparations maintained in twin-compartment boxes

\begin{tabular}{lll}
\hline & \multicolumn{2}{l}{$\begin{array}{l}\text { Glucose concentration } \\
(\mathrm{mmol} / \mathrm{l})\end{array}$} \\
\cline { 2 - 3 } & $\begin{array}{l}5.5 \\
(n=5)\end{array}$ & $\begin{array}{l}25.0 \\
(n=6)\end{array}$ \\
\hline $\begin{array}{l}\text { Incorporated }{ }^{3} \mathrm{H} \text { fucose } \\
\text { (as DPM } / \mu \mathrm{Ci} \text { added) }\end{array}$ & $367 \pm 23^{\mathrm{a}}$ & $257 \pm 38^{\mathrm{a}}$ \\
$\begin{array}{l}\text { Incorporated }{ }^{14} \mathrm{C} \text { leucine } \\
\text { (as DPM } / \mu \mathrm{Ci} \text { added) }\end{array}$ & $109 \pm 4$ & $98 \pm 12$ \\
Noradrenaline (pmol/nerve) & $334 \pm 31$ & $320 \pm 27$ \\
\hline
\end{tabular}

Net accumulations of incorporated fucose, leucine and noradrenaline in five control preparations and six preparations (from different animals) incubated with the nerves exposed to $25 \mathrm{mmol} / 1$ glucose. DPM $=$ disintegrations per minute. Values expressed as mean \pm SEM; a $p<0.05$ by unpaired t-test

\section{Calculations of Net Accumulations}

Accumulation of all materials measured was confined to the $3 \mathrm{~mm}$ of nerve immediately proximal to the constriction. The background radioactivity and noradrenaline content were estimated from the segments taken between points $5-10 \mathrm{~mm}$ proximal to the ligature. Disintegrations per min values for both isotopes and noradrenaline contents were calculated as equivalents for $3-\mathrm{mm}$ nerve in this region and the data subtracted from the sum of the values for the $3-\mathrm{mm}$ nerve immediately proximal to the constriction. The segments of nerve used to calculate background data were also confined in the 'nerve trunk' compartments of the culture chambers.

\section{Statistical Analysis}

All the results are presented as mean \pm SEM. Comparison of data between different preparations, incubated in twin compartment chambers, was made using unpaired t-tests. Comparisons of accumulations in the two nerves of single preparations, incubated in three compartment chambers, was made using paired t-tests.

\section{Results}

\section{Effects of 25 mmol/l Glucose on Accumulation in Two Compartment Chambers}

The net accumulations of incorporated fucose, incorporated leucine and noradrenaline in nerves incubated in normal and elevated extracellular glucose are shown in Table 1. The distribution of these accumulations between the individual 1-mm nerve segments proximal to the ligatures are shown in Figure 2.

These data show a statistically significant reduction in the accumulation of incorporated fucose in the nerves of those preparations exposed to $25 \mathrm{mmol} / 1 \mathrm{glu}-$ cose. The bulk of the deficit of fucose accumulation in the high glucose-treated preparations was seen in segments $P_{1}$ and $P_{2}$ (Fig. 2). The amounts of fucose further away from the ligature were similar in the nerves of both control and experimental preparations.

The apparent reductions in accumulation of incorporated leucine and of endogenous noradrenaline in

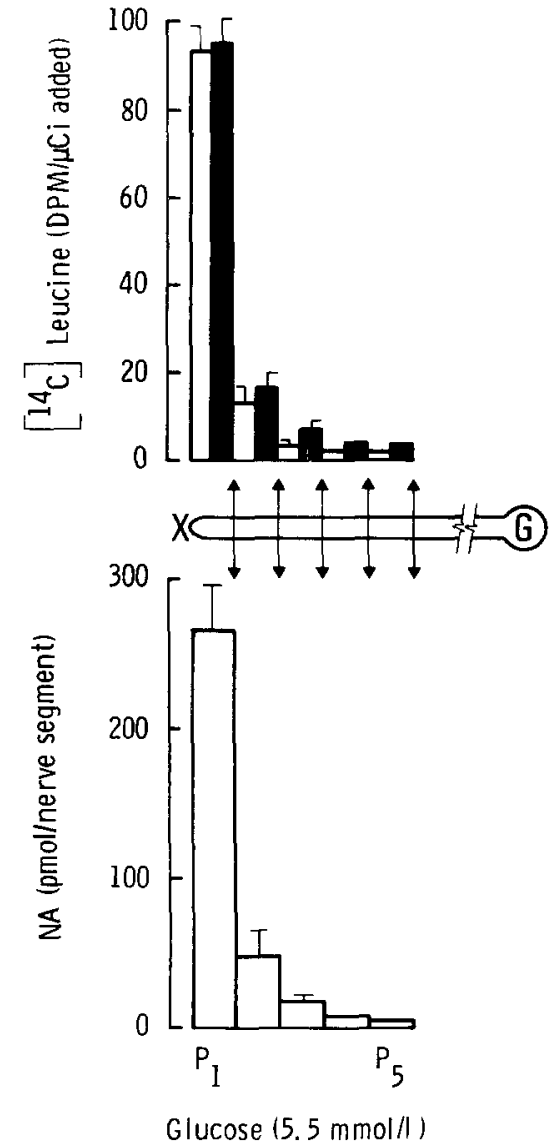

Glucose $(5.5 \mathrm{mmol} / \mathrm{l})$
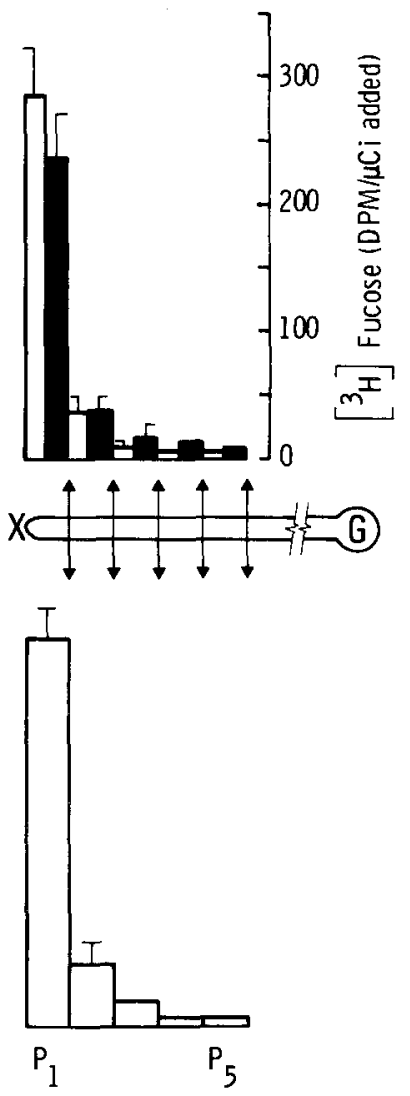

Glucose $(25 \mathrm{mmol} / \mathrm{l})$
Fig. 2. The distribution of incorporated fucose (ם), incorporated leucine ( $\square$ ) (upper panel) and noradrenaline (NA) (lower panel) between 1-mm segments proximal to ligatures applied to hypogastric nerves of preparations maintained in twincompartment chambers. The nerve trunks were bathed in either $5.5 \mathrm{mmol} / 1$ glucose $(n=5)$ or $25 \mathrm{mmol} / 1$ glucose $(n=6)$. $G$ : inferior mesenteric ganglion. Values expressed as mean + SEM. The net accumulations of material calculated from these data are shown in Table 1 


\begin{tabular}{|c|c|c|c|}
\hline Glucose $(\mathrm{mmol} / \mathrm{l})$ & 5.5 & 5.5 & 25.0 \\
\hline $\begin{array}{l}\text { Incorporated fucose } \\
\text { (DPM/ } / \mu C i \text { ) }\end{array}$ & $378-34$ & $p<0.001$ & $274 \pm 36$ \\
\hline $\begin{array}{l}\text { Incorporated leucine } \\
\text { (DPM//Ci) }\end{array}$ & $146 \pm 20$ & $p<0.01$ & $118 \pm 24$ \\
\hline $\begin{array}{l}\text { Noradrenaline } \\
\text { (pmol) }\end{array}$ & $326-24$ & NS & $338-28$ \\
\hline
\end{tabular}

Fig. 3. Net accumulations of incorporated fucose, incorporated leucine and of noradrenaline proximal to constrictions applied to hypogastric nerves of preparations maintained in three-compartment culture chambers. Values expressed as mean $\pm S E M$ for five preparations.

preparations treated with elevated extracellular glucose did not attain statistical significance by comparison with control accumulations.

The incorporation of both isotopes and the content of noradrenaline in the ganglia of both groups of preparations were similar (see below). This indicates that exposure of the axons to $25 \mathrm{mmol} / 1$ glucose did not alter protein, glycoprotein or catecholamine synthesis in their cell bodies. The latter were maintained in normal extracellular glucose on the other side of the barrier.

The mean glucose concentrations in the media at the end of the experiments did not differ by $>2 \%$ from the amounts dissolved at the start.

\section{Effects of $25 \mathrm{mmol} / \mathrm{l}$ Glucose in Three Compartment Chambers}

The net accumulations of incorporated fucose, incorporated leucine and noradrenaline in experiments with the three compartment chambers are shown in Figure 3. The distribution of these materials between the segments of nerve proximal to the ligature was similar to that seen in the earlier experiments. This has therefore not been illustrated.

The accumulations of fucose, leucine and noradrenaline in the nerves maintained in a normal glucose concentration were similar to those seen in the control experiments using the twin compartment chambers. Again, exposure to glucose at $25 \mathrm{mmol} / 1$ caused a marked and significant reduction in the accumulation of incorporated fucose. However, the improved experimental design offered by the three compartment chamber gave a statistically significant reduction in the accumulation of incorporated leucine. There was, however, no effect of glucose elevation on noradrenaline accumulation.

Incorporation of the isotopes in the ganglia and the noradrenaline content of the latter were similar to those seen in the two compartment experiments (Fig. 3).

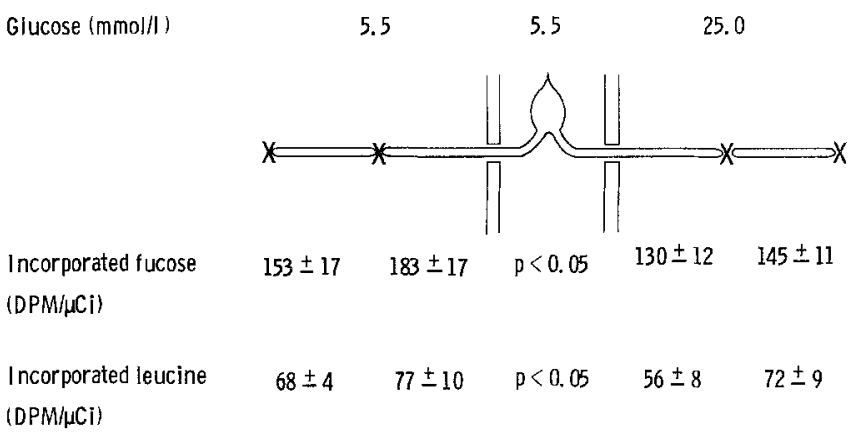

Fig.4. Net accumulations of incorporated fucose and leucine proximal to double ligations applied asynchronously to hypogastric nerves of preparations maintained in three compartment chambers. For the first $24 \mathrm{~h}$ incubation the glucose concentration in all three compartments was $5.5 \mathrm{mmol} / \mathrm{l}$. The second (more proximal) ligatures were then tied on each nerve and the glucose concentration in the right hand nerve compartment was increased to $25 \mathrm{mmol} / 1$ for a further $24-h$ incubation. Values expressed as mean \pm SEM for four preparations. Values indicate the significance of difference between the mean accumulations against the more proximal ligatures (paired $t$-tests between contralateral nerves)

Table 2. The effects of elevated extracellular glucose in isotonic medium

\begin{tabular}{|c|c|c|c|}
\hline & $\begin{array}{l}\text { Nerve } \\
\text { compartment A }\end{array}$ & $\begin{array}{l}\text { Ganglion } \\
\text { compartment }\end{array}$ & $\begin{array}{l}\text { Nerve } \\
\text { compartment } \mathbf{B}\end{array}$ \\
\hline & \multicolumn{3}{|c|}{ Glucose concentration (mmol/l) } \\
\hline & 5.5 & 5.5 & 25.0 \\
\hline $\begin{array}{l}\text { Accumulation } \\
\text { of incorporated } \\
{ }^{3} \mathrm{H} \text { fucose } \\
\text { (DPM/ } / \mu \mathrm{Ci} \\
\text { added) }\end{array}$ & $350 \pm 41$ & $p<0.01$ & $287 \pm 21$ \\
\hline $\begin{array}{l}\text { Accumulation } \\
\text { of incorporated } \\
{ }^{14} \mathrm{C} \text { leucine } \\
\text { (DPM } / \mu \mathrm{Ci} \\
\text { added) }\end{array}$ & $161 \pm 19$ & $p<0.01$ & $130 \pm 19$ \\
\hline
\end{tabular}

Net accumulations of incorporated fucose and leucine in four preparations maintained in three-compartment chambers. The nerve trunks were exposed to either normal culture medium (compartment $A$ and ganglion compartment) or medium with increased glucose plus water to 300 mosmol. DPM = disintegrations per min. Values expressed as mean \pm SEM

Glucose concentrations in the media at the end of the experiments were all within $2 \%$ of the starting values.

\section{Asynchronous Double Ligation Experiments}

The results of these experiments are shown in Figure 4. The data show that $25 \mathrm{mmol} / 1$ glucose did not reduce the content of incorporated fucose and leucine which had accumulated during the first $24-\mathrm{h}$ incubation in $5.5 \mathrm{mmol} / \mathrm{l}$ glucose and had been isolated between the 
first and second ligatures. However, the accumulation of incorporated fucose and leucine, against the second (more proximal) ligature (which was transported towards that ligature under conditions of $25 \mathrm{mmol} / 1 \mathrm{glu}-$ cose), was significantly reduced by comparison with the accumulation in the contralateral hypogastric nerves, which were exposed to $5.5 \mathrm{mmol} / 1$ glucose throughout the whole experiment.

\section{Effects of Elevated Glucose in Isotonic Media}

A small number of experiments were performed to distinguish between the effects of elevation of the tonicity of the medium due to addition of glucose and the chemical effects of increased extracellular glucose per se. The results are shown in Table 2. These show that the reductions in accumulation of incorporated fucose and leucine were still seen when $25 \mathrm{mmol} / 1$ glucose was present in an isotonic medium.

\section{Soluble Radioactivity in the Nerve Segment Extracts}

A small aliquot of each perchlorate extract was taken for scintillation counting prior to noradrenaline assay (see Methods). In experiments using both two and three compartment chambers there was an accumulation of extracted soluble radioactivity. The amounts, however, were uniformly small compared with the insoluble activity. For the series of experiments using three compartment chambers, the net accumulations of soluble activity (expressed as disintegrations per minute $/ \mu \mathrm{Ci}$ added isotope) in the nerves exposed to $5.5 \mathrm{mmol} / 1 \mathrm{glu}-$ cose were $4.6 \pm 0.1\left({ }^{3} \mathrm{H}\right)$ and $0.44 \pm 0.04\left({ }^{14} \mathrm{C}\right)$. For the contralateral nerves, exposed to $25 \mathrm{mmol} / \mathrm{l}$, the accumulations were $5.00 \pm 0.3\left({ }^{3} \mathrm{H}\right)$ and $0.40 \pm 0.07\left({ }^{14} \mathrm{C}\right)$. No significant difference between the two conditions was revealed by paired t-test. The experiments using twin compartment chambers gave similar results.

\section{Incorporated Radioactivity in Ganglia}

At the end of all experiments the incorporated (perchlorate-precipitated) radioactivity in the ganglion was measured and calculated with reference to ganglia weight and the amount of added isotope (see Methods). This activity is therefore expressed as disintegrations per minute/mg tissue $/ \mu \mathrm{Ci}$ added isotope. For preparations using the twin compartment chambers the activities were, for $5.5 \mathrm{mmol} / 1$ glucose (nerve compartment), $864 \pm 30\left({ }^{3} \mathrm{H}\right)$ and $858 \pm 16\left({ }^{14} \mathrm{C}\right)$ and for $25 \mathrm{mmol} / 1 \mathrm{glu}-$ cose (nerve compartment), $868 \pm 32\left({ }^{3} \mathrm{H}\right)$ and $918 \pm 37$ $\left({ }^{14} \mathrm{C}\right)$. There were no differences between conditions (glucose concentration) for either isotope. For preparations incubated in the three compartment chambers the activities were $898 \pm 25\left({ }^{3} \mathrm{H}\right)$ and $899 \pm 31\left({ }^{14} \mathrm{C}\right)$ disintegrations per minute/mg tissue $/ \mu \mathrm{Ci}$ added isotope.

\section{Discussion}

The experiments described here show that incubation of cat hypogastric nerves in a culture medium containing $25 \mathrm{mmol} / 1$ glucose reduced the accumulation of incorporated fucose and leucine proximal to ligatures applied to the nerve trunks. Incorporation of the isotopes in the ganglia occurred in media of normal glucose concentration and the acid-precipitated radioactivity in the ganglion was similar in all experiments. This indicates that, of the total radioactivity, the fraction which was present in macromolecules (acid-precipitated) was unaltered by exposure to high glucose. Hence it may be argued that the reduced accumulation of material under conditions of high glucose does not reflect reduced incorporation of the labelled tracers into macromolecules.

A second consideration is the possibility that elevation of extracellular glucose in the fluid bathing the nerve trunks caused a loss of label from the macromolecules within the axons. This possibility was examined by the double ligation experiments, when it was found that material, which has already accumulated at "normal' glucose concentration, was unaffected by elevation of the glucose to $25 \mathrm{mmol} / \mathrm{l}$. This refutes the possibility that the increased glucose concentration caused a loss of isotope from macromolecules within the axon. This conclusion is further supported by the fact that the levels of radioactivity extracted from the nerve segments proximal to the ligatures by the perchlorate were insignificant and unaffected by high glucose. This indicates that there was relatively little turnover of fucose and leucine in the axons irrespective of the glucose concentration.

This attempt to mimic hyperglycaemia in vitro poses problems in the design of control experiments to distinguish between the effects of an increase in the concentration of glucose and the consequent increase in osmolality of the extracellular fluid. In the control experiments water was added with the excess glucose to maintain normal osmolality. This approach brings problems of validity due to the inevitable dilution of the other components of the culture medium. However, this dilution is of the order of only $5.8 \%$ and this, taken together with the finding that the reduced accumulation of macromolecules was similar in nerves exposed to high glucose in either hyper- or normotonic media, indicates strongly that the glucose, rather than the hypertonicity was the causative factor. Questions of control aside, the addition of glucose to normal media may be judged as valid by reference to studies on experimental diabetes in rats. These animals show hyperglycaemia as pronounced as $25 \mathrm{mmol} / 1$ which co-exists with volaemic expansion, slight hyponatraemia [4] and an increase in plasma osmolality of about $20 \mathrm{mosmol}$ (S. M. Gardiner and T. Bennett, unpublished observations).

The above considerations lead to the suggestion that the principal effect of the $25 \mathrm{mmol} / 1$ glucose media was to reduce the axonal transport of macromolecules con- 
taining leucine and fucose. The present experiments do not, however, permit distinction to be made between the amount of material transported and the rate of transport. There are no published accounts of the velocity components which comprise anterograde transport in cat hypogastric nerves. Such studies have begun recently in our laboratories and two components of orthograde transport have been identified. The fastest $(200-250 \mathrm{~mm} /$ day) contains protein, glycoprotein and noradrenaline; this is followed by a slower peak of protein and glycoprotein with no noradrenaline $(25-30 \mathrm{~mm} /$ day). The studies reported in this paper promote the hypothesis that the slower component may be affected selectively by elevated extracellular glucose.

An alternative suggestion, to explain the disparity of effects on noradrenaline accumulation and that of labelled macromolecules, is that different types of fibre participate in the response to elevated extracellular glucose. The hypogastric nerves of the cat comprise about $85 \%$ non-myelinated fibres, a large proportion of which contain accumulated noradrenaline after constriction $[12,13,22]$. The remainder are myelinated fibres which would not be expected to arise from cell bodies in the inferior mesenteric ganglion since decentralisation of the ganglion causes these fibres to degenerate [22]. It is therefore likely that the accumulations studied here occurred in non-myelinated axons, though some fraction of these may not have been noradrenergic. In this context it is interesting to note that Jakobsen has reported indications that experimental diabetes may promote disordered anterograde transport in non-myelinated, but not in myelinated, fibres of the same nerve trunk [8].

If the finding, that elevated extracellular glucose impairs anterograde transport in hypogastric nerves, is relevant to diabetes it must relate to published studies on axonal transport in diabetic animals. The extensive work of Jakobsen and Sidenius on sensory neurones of rats reveals that defects of retrograde transport are present in experimental diabetes [8-11, 19-21]. However, these authors found no defects of fast orthograde transport in dorsal root afferents, though a defect of slow orthograde transport was discovered [11]. Other studies have also shown that orthograde transport in sensory neurones is unaffected in experimental diabetes $[1,3]$. In contrast motor fibres have been found to exhibit slowed or reduced orthograde axonal transport in diabetic animals [14-16, 18, 24]. The possibility exists, therefore, that axonal transport in different divisions of the peripheral nervous system is not uniformly affected by experimental diabetes. There is little work on the phenomenon in autonomic efferents, although the present finding of normal orthograde noradrenaline transport in high glucose media agrees well with a similar result in streptozotocin-diabetic rats [23].

Most of the studies referred to above have shown that impairments of axonal transport in experimental diabetes are ameliorated by insulin therapy. Thus, the present findings, together with studies in vivo, give strong support to the premise that hyperglycaemia per se may be a prime cause of disordered axonal transport in experimental diabetes.

Acknowledgements. I am indebted to the following people: $\mathrm{Mr}$ I. Topham for technical assistance, Mr. S. Henshaw for construction of the culture chambers and Mr. G. Errington and Miss S. Ecob for their meticulous and humane care of the animals. Financial assistance from ICI, Pharmaceuticals Division together with the personal support and interest of Dr. D.C. N. Earl is gratefully acknowledged.

\section{References}

1. Bajada A, Sharma AK, Thomas PK (1980) Axoplasmic transport in vagal afferent fibres in normal and alloxan-diabetic rabbits. $J$ Neurol Sci 47: 365-378

2. Banks P, Mayor D, Mitchell M, Tomlinson DR (1971) Studies on the translocation of noradrenaline-containing vesicles in postganglionic sympathetic neurones in vitro. Inhibition of movement by colchicine and vinblastine and evidence for the involvement of axonal microtubules. J Physiol 216: 625-639

3. Bisby MA (1980) Axonal transpot of labelled protein and regeneration rate in nerves of streptozotocin-diabetic rats. Exp Neurol 69: 74-84

4. Christlieb AR, Long R, Underwood RH (1979) Renin-angiotensin-aldosterone system, electrolyte homeostasis and blood pressure in alloxan diabetes. Am J Med Sci 277: 295-303

5. Cuenod M, Sandri C, Akert K (1972) Enlarged synaptic vesicles in optic nerve terminals induced by intraocular injection of colchicine. Brain Res 39: 285-296

6. Eagle H (1959) Amino acid metabolism in mammalian cell cultures. Science 130: 432-437

7. Green JH, Macdonald IA (1981) The influence of intravenous glucose on body temperature. Quart J Exp Physiol 66: 465-473

8. Jakobsen J (1979) Early and preventable changes of peripheral nerve structure and function in insulin-deficient diabetic rats. $J$ Neurol Neurosurg Psychiatr 42: 509-518

9. Jakobsen J, Brimijoin S, Skau K, Sidenius P, Wells D (1981) Retrograde axonal transport of transmitter enzymes, fucose-labelled protein, and nerve growth factor in streptozocin diabetic rats. Diabetes 30: 797-803

10. Jakobsen J, Sidenius P (1979) Decreased axonal flux of retrogradely transported glycoproteins in early experimental diabetes. J Neurochem 33: 1055-1060

11. Jakobsen J, Sidenius P (1980) Decreased axonal transport of structural proteins in streptozotocin diabetic rats. J Clin Invest 66 : 292-297

12. Kapeller K, Mayor D (1969) An electron microscopic study of the early changes proximal to a constriction in sympathetic nerves. Proc Roy Soc B 172: 39-51

13. Mayor D, Kapeller K (1967) Fluorescence microscopy and electron microscopy of adrenergic nerves after constriction at two points. J Roy Microscopical Soc 87: 277-294

14. McLean WG, Meiri KF (1980) Rapid axonal transport of protein in rat sciatic motor nerves during early experimental diabetes. $J$ Physiol 301: 43-44 P (Abstract)

15. McLean WG, Meiri KF (1981) Slow axonal transport of protein in rat sciatic motor nerves during early experimental diabetes. J Physiol 310: 57 P (Abstract)

16. Meiri KF, McLean WG (1982) Axonal transport of protein in motor fibres of experimentally diabetic rats - fast anterograde transport. Brain Res 238: 77-88

17. Perisic M, Cuenod M (1972) Synaptic transmission depressed by colchicine blockade of axoplasmic flow. Science 175: 1140-1142

18. Schmidt RE, Matschinsky FM, Godfrey DA, Williams AD, McDougal DB (1975) Fast and slow axoplasmic flow in sciatic nerve of diabetic rats. Diabetes 24: 1081-1085 
19. Sidenius P, Jakobsen J (1979) Axonal transport in early experimental diabetes. Brain Res 173: 315-330

20. Sidenius $P$, Jakobsen J (1980) Impaired retrograde axonal transport from a nerve crush in streptozotocin diabetic rats. Diabetologia 19:222-228

21. Sidenius P, Jakobsen J (1981) Retrograde axonal transport: a possible role in the development of neuropathy. Diabetologia 20: $110-112$

22. Tomlinson DR (1972) Intra-axonal storage and transport of noradrenaline in post-ganglionic noradrenergic nerves. $\mathrm{Ph} D$ Thesis: University of Sheffield

23. Tomlinson DR, Gillon KRW, Smith MG (1982) Axonal transport of noradrenaline and noradrenergic transmission in rats with streptozotocin-induced diabetes. Diabetologia 22: 199-204
24. Tomlinson DR, Holmes PR, Mayer JH (1982) Reversal, by treatment with an aldose reductase inhibitor, of impaired axonal transport and motor nerve conduction velocity in experimental diabetes mellitus. Neurosci Lett 31: 189-193

Received: 17 February 1982

and in revised form: 5 October 1982

Dr. D.R. Tomlinson

Department of Physiology and Pharmacology Medical School

Queen's Medical Centre

Nottingham $\mathrm{NG} 72 \mathrm{UH}$

UK 